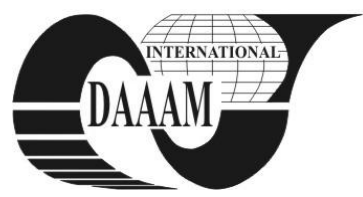

\title{
DYNAMIC COEFFICIENTS DUE TO THE ANGULAR DISPLACEMENTS OF THE SHAFT
}

\author{
PARAUSANU, I[oan]; PETRE, C[ristian]; TUDOSE, V[irgil] \& GHEORGHIU, H[oria]
}

\begin{abstract}
In order to improve the calculation of the critical rotor speed by including the flexibility of the bearing oil film due to the angular displacements of the shaft, the load capacity, total moment, stiffness and damping coefficients are obtained using a numerical method.
\end{abstract}

Key words: journal bearings, stiffness and damping coefficients

\section{INTRODUCTION}

From the unaligned journal bearings the pressure distribution is much different in comparison with the aligned case, becoming strongly non-symmetric, so it is obvious that this pressure produces not only a load capacity, but a moment too. This must be taken into account when calculating the stiffness and damping coefficients of unaligned journal bearings. The angular displacements and velocities produce strong effects on the moment attitude angle. The pressure distribution law was obtained by solving the Reynolds equation in the case of a conical motion of the shaft in a journal bearing (Parausanu et al., 2010). In order to improve the calculation of the critical rotor speed by including the flexibility of the bearing oil film.

\section{NOMENCLATURE}

$J$ - diametral clearance;

$n$ - shaft speed;

$x, y, z$ - cartesian co-ordinates (fixed $x O y z$ - frame);

$X, Y, Z$ - cartesian co-ordinates (rotated $X O Y Z$ - frame);

$\alpha, \beta$ - angular displacements;

$\dot{\alpha}, \dot{\beta}$ - angular velocities;

$\dot{\alpha}^{*}, \dot{\beta}^{*}$ - dimensionless angular velocities, $(\dot{\alpha}, \dot{\beta}) /(\pi \cdot n)$;

$\varepsilon \quad$ - eccentricity ratio, $2 e / J$;

$\Psi$ - dimensionless clearance, $J / D$;

$\eta$ - dynamic viscosity;

$\theta$ - angular co-ordinate.

\section{CALCULATION OF THE TOTAL LOAD}

Integrating the pressure distribution $p$ (Parausanu et al., 2010) on the friction area the components of the forces and moments are obtained in the matriceal form:

$$
\left[\begin{array}{c}
F_{x}^{\prime} \\
F_{y}^{\prime} \\
M_{x}^{\prime} \\
M_{y}^{\prime}
\end{array}\right]=\frac{1}{2} \int_{A}(p+|p|)\left[\begin{array}{c}
\cos \theta^{\prime} \\
\sin \theta^{\prime} \\
-z \sin \theta^{\prime} \\
z \cos \theta^{\prime}
\end{array}\right] \mathrm{d} A=\frac{3 \pi}{4} C_{p} F_{0}\left[\begin{array}{c}
J_{1} \\
J_{2} \\
\frac{D}{2} J_{3} \\
\frac{D}{2} J_{4}
\end{array}\right]
$$

where $C_{p}$ is the load capacity coeffcient:

$$
C_{p}=\frac{n \eta}{p_{m} \Psi^{2}}
$$

and the integrals $J_{1}, J_{2}, J_{3}$ and $J_{4}$ are:

$$
\left[\begin{array}{l}
J_{1} \\
J_{2} \\
J_{3} \\
J_{4}
\end{array}\right]=\frac{1}{2} \int_{-1}^{1} \int_{0}^{2 \pi}(\bar{p}+|\bar{p}|) \cdot\left[\begin{array}{c}
\cos \theta^{\prime} \\
\sin \theta^{\prime} \\
-\bar{z} \sin \theta^{\prime} \\
\bar{z} \cos \theta^{\prime}
\end{array}\right] \cdot \mathrm{d} \theta^{\prime} \cdot \mathrm{d} \bar{z}
$$

In the $x O y z$ - frame the total loads are

$$
\left[\begin{array}{c}
F_{x} \\
F_{y} \\
M_{x} \\
M_{y}
\end{array}\right]=\left[\begin{array}{cc}
{[\mathrm{T}]} & {[0]} \\
{[0]} & {[\mathrm{T}]}
\end{array}\right] \cdot\left[\begin{array}{c}
F_{x}^{\prime} \\
F_{y}^{\prime} \\
M_{x}^{\prime} \\
M_{y}^{\prime}
\end{array}\right]
$$

where [0] is the zero matrice $(2 \times 2)$ and $[\mathrm{T}]$ is the transfer coordinates matrice.

\section{CALCULATION OF DYNAMIC COEFFICIENTS}

In the journal bearing, the force vector can be written:

where:

$$
\{F\}=\left\{F_{0}\right\}+[\mathrm{K}]\{x\}+[\mathrm{C}]\{\dot{x}\}
$$

$$
\{F\}=\left[\begin{array}{llll}
F_{x} & F_{y} & M_{x} & M_{y}
\end{array}\right]^{T} ;\left\{F_{0}\right\}=\left[\begin{array}{llll}
F_{0} & 0 & 0 & 0
\end{array}\right]^{T}
$$

and $F_{0}$ is the static load along the $O x$ axis, and

$$
\{x\}=\left[\begin{array}{llll}
x & y & \alpha & \beta
\end{array}\right]^{T} ;\{\dot{x}\}=\left[\begin{array}{llll}
\dot{x} & \dot{y} & \dot{\alpha} & \dot{\beta}
\end{array}\right]^{T}
$$

are the displacement and the velocity vectors of the centre of the journal in the fixed $x O y z$ - frame.

$$
[\mathrm{K}]=\left[\begin{array}{cccc}
k_{x x} & k_{x y} & k_{x \alpha} & k_{x \beta} \\
k_{y x} & k_{y y} & k_{y \alpha} & k_{y \beta} \\
k_{\alpha x} & k_{\alpha y} & k_{\alpha \alpha} & k_{\alpha \beta} \\
k_{\beta x} & k_{\beta y} & k_{\beta \alpha} & k_{\beta \beta}
\end{array}\right]=\left[\begin{array}{c}
\partial F_{x} \\
\partial F_{y} \\
\partial M_{x} \\
\partial M_{y}
\end{array}\right]\left[\begin{array}{llll}
\frac{1}{\partial x} & \frac{1}{\partial y} & \frac{1}{\partial \alpha} & \frac{1}{\partial \beta}
\end{array}\right]
$$

The terms of the stiffness and damping matrices are presented in equations (8) and (9).

The relationships between differential operators in the two cartesian systems, fixed and mobile are presented in equations (10).

$$
[\mathrm{C}]=\left[\begin{array}{cccc}
c_{x x} & c_{x y} & c_{x \alpha} & c_{x \beta} \\
c_{y x} & c_{y y} & c_{y \alpha} & c_{y \beta} \\
c_{\alpha x} & c_{\alpha y} & c_{\alpha \alpha} & c_{\alpha \beta} \\
c_{\beta x} & c_{\beta y} & c_{\beta \alpha} & c_{\beta \beta}
\end{array}\right]=\left[\begin{array}{c}
\partial F_{x} \\
\partial F_{y} \\
\partial M_{x} \\
\partial M_{y}
\end{array}\right]\left[\begin{array}{llll}
\frac{1}{\partial \dot{x}} & \frac{1}{\partial \dot{y}} & \frac{1}{\partial \dot{\alpha}} & \frac{1}{\partial \dot{\beta}}
\end{array}\right]
$$




$$
\left[\begin{array}{l}
\frac{\partial}{\partial x} \\
\frac{\partial}{\partial y} \\
\frac{\partial}{\partial \alpha} \\
\frac{\partial}{\partial \beta}
\end{array}\right]=\left[\begin{array}{ll}
\frac{2}{J}[\mathrm{~T}] & {[0]} \\
{[0]} & {[\mathrm{T}]}
\end{array}\right] \cdot\left[\begin{array}{c}
\frac{\partial}{\partial \varepsilon} \\
\frac{1}{\varepsilon} \frac{\partial}{\partial \phi} \\
\frac{\partial}{\partial \alpha^{\prime}} \\
\frac{\partial}{\partial \beta^{\prime}}
\end{array}\right] ;\left[\begin{array}{c}
\frac{\partial}{\partial \dot{x}} \\
\frac{\partial}{\partial \dot{y}} \\
\frac{\partial}{\partial \dot{\alpha}} \\
\frac{\partial}{\partial \dot{\beta}}
\end{array}\right]=\frac{1}{\pi \cdot n}\left[\begin{array}{cc}
\frac{2}{J}[\mathrm{~T}] & {[0]} \\
{[0]} & {[\mathrm{T}]}
\end{array}\right] \cdot\left[\begin{array}{c}
\frac{\partial}{\partial \varepsilon^{*}} \\
\frac{1}{\varepsilon} \frac{\partial}{\partial \phi^{*}} \\
\frac{\partial}{\partial \alpha^{*}} \\
\frac{\partial}{\partial \beta^{*}}
\end{array}\right]
$$

Finally, introducing equations (1) and (3) in (8) and (9) and taking into account the relationships (10) result:

$$
\begin{aligned}
& {\left[\begin{array}{llll}
k_{x x} & k_{x y} & k_{y x} & k_{y y}
\end{array}\right]^{T}=\frac{3 \pi}{2} C_{p} \frac{F_{0}}{J}\left[\mathrm{R}_{1}\right] .}
\end{aligned}
$$

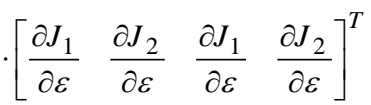

$$
\begin{aligned}
& {\left[\begin{array}{llll}
k_{\alpha x} & k_{\alpha y} & k_{\beta x} & k_{\beta y}
\end{array}\right]^{T}=\frac{3 \pi}{4} C_{p} \frac{F_{0}}{\Psi}\left[\mathrm{R}_{1}\right] .} \\
& \cdot\left[\begin{array}{llll}
\frac{\partial J_{3}}{\partial \varepsilon} & \frac{\partial J_{4}}{\partial \varepsilon} & \frac{\partial J_{3}}{\partial \varepsilon} & \frac{\partial J_{4}}{\partial \varepsilon}
\end{array}\right]^{T} \\
& {\left[\begin{array}{llll}
k_{x \alpha} & k_{x \beta} & k_{y \alpha} & k_{y \beta}
\end{array}\right]^{T}=\frac{3 \pi}{4} C_{p} F_{0}\left[\mathrm{R}_{2}\right] .} \\
& \cdot\left[\begin{array}{llll}
\frac{\partial J_{1}}{\partial \alpha^{\prime}} & \frac{\partial J_{1}}{\partial \beta^{\prime}} & \frac{\partial J_{2}}{\partial \alpha^{\prime}} & \frac{\partial J_{2}}{\partial \beta^{\prime}}
\end{array}\right]^{T} \\
& {\left[\begin{array}{llll}
k_{\alpha \alpha} & k_{\alpha \beta} & k_{\beta \alpha} & k_{\beta \beta}
\end{array}\right]^{T}=\frac{3 \pi}{8} C_{p} F_{0} D\left[\mathrm{R}_{2}\right] .} \\
& \cdot\left[\begin{array}{llll}
\frac{\partial J_{3}}{\partial \alpha^{\prime}} & \frac{\partial J_{3}}{\partial \beta^{\prime}} & \frac{\partial J_{4}}{\partial \alpha^{\prime}} & \frac{\partial J_{4}}{\partial \beta^{\prime}}
\end{array}\right]^{T} \\
& {\left[\begin{array}{llll}
c_{x x} & c_{x y} & c_{y x} & c_{y y}
\end{array}\right]^{T}=\frac{3}{2} C_{p} \frac{F_{0}}{n \cdot J}\left[\mathrm{R}_{2}\right] .} \\
& \cdot\left[\frac{\partial J_{1}}{\partial \varepsilon^{*}} \frac{1}{\varepsilon} \frac{\partial J_{1}}{\partial \phi^{*}} \frac{\partial J_{2}}{\partial \varepsilon^{*}} \frac{1}{\varepsilon} \frac{\partial J_{2}}{\partial \phi^{*}}\right]^{T} \\
& {\left[\begin{array}{cccc}
c_{\alpha x} & c_{\alpha y} & c_{\beta x} & c_{\beta y}
\end{array}\right]^{T}=\frac{3}{4} C_{p} \frac{F_{0}}{n \cdot \Psi}\left[\mathrm{R}_{2}\right] .} \\
& \cdot\left[\frac{\partial J_{3}}{\partial \varepsilon^{*}} \frac{1}{\varepsilon} \frac{\partial J_{3}}{\partial \phi^{*}} \frac{\partial J_{4}}{\partial \varepsilon^{*}} \frac{1}{\varepsilon} \frac{\partial J_{4}}{\partial \phi^{*}}\right]^{T} \\
& {\left[\begin{array}{cccc}
c_{x \alpha} & c_{x \beta} & c_{y \alpha} & c_{y \beta}
\end{array}\right]^{T}=\frac{3}{4} C_{p} \frac{F_{0}}{n}\left[\mathrm{R}_{2}\right] .} \\
& \cdot\left[\frac{\partial J_{1}}{\partial \alpha^{*}} \frac{\partial J_{1}}{\partial \beta^{*}} \frac{\partial J_{2}}{\partial \alpha^{*}} \frac{\partial J_{2}}{\partial \beta^{*}}\right]^{T} \\
& {\left[\begin{array}{cccc}
c_{\alpha \alpha} & c_{\alpha \beta} & c_{\beta \alpha} & c_{\beta \beta}
\end{array}\right]^{T}=\frac{3}{8} C_{p} \frac{F_{0} D}{n}\left[\mathrm{R}_{2}\right]} \\
& \cdot\left[\begin{array}{llll}
\frac{\partial J_{3}}{\partial \alpha^{*}} & \frac{\partial J_{3}}{\partial \beta^{*}} & \frac{\partial J_{4}}{\partial \alpha^{*}} & \frac{\partial J_{4}}{\partial \beta^{*}}
\end{array}\right]^{T}
\end{aligned}
$$

where the matrices $\left[R_{1}\right]$ and $\left[R_{2}\right]$ are $4 \times 4$ matrices in $\sin \phi$ and $\cos \phi$. After a numerical resolution of the integrals of the equations (11)-(18), one can obtain the dynamic coefficents, which are presented in Figure 1.
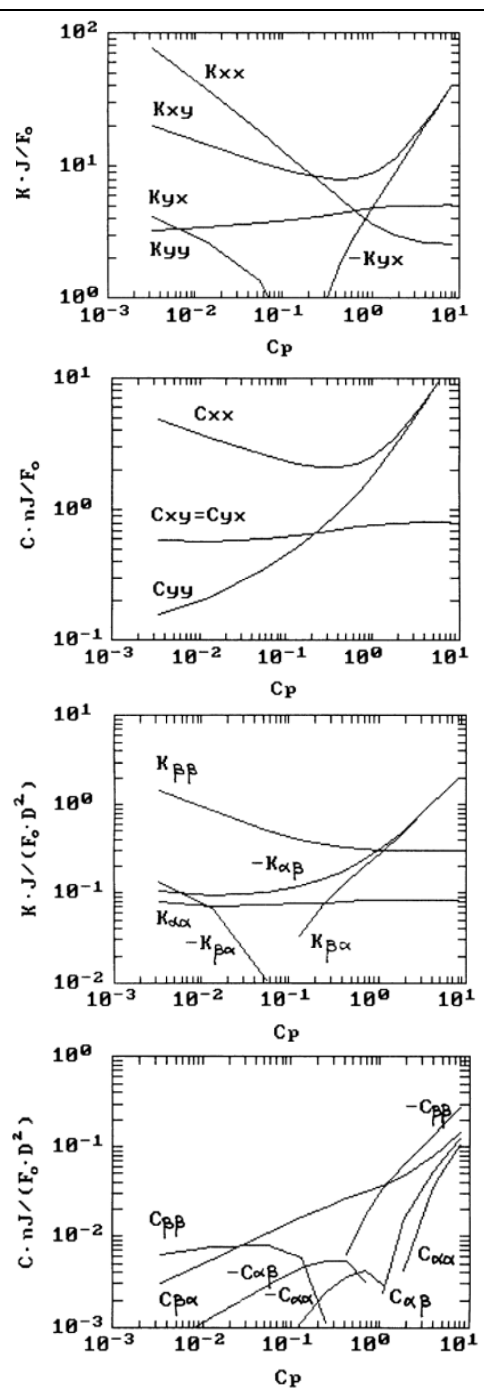

Fig. 1. Dynamic coefficients of unaligned journal bearing

\section{CONCLUSIONS}

The 8 coefficients associated with a uniform lateral eccentricity of the shaft are the same that those of the classical aligned case, so the model boundary verifications are satisfied.

The other 8 coefficients obtained improve the calculation of the critical speed of a rotor by including the flexibility of the bearing oil film due to angular displacements of the shaft.

\section{REFERENCES}

Baskharone, E.A. \& Hensel, S.J. (1991). Interrelated Rotordynamic Effects of Cylindrical and Conical Whirl of Annular Seal Rotors, ASME, Journal of Tribology, vol. 113, pp. $470-480$

Kanemory, Y., Iwatsubo, T. (1992). Experimental Study of Dynamic Fluid Forces and Moments of a Long Annular Seal, Journal of Tribology, vol. 114, 2, pp. 773-778

Parausanu, I.; Petre, C.; Gheorghiu, H. \& Vlasceanu, D. (2010). The Oil Pressure Law in Journal Bearings Due to of the Shaft Conical Motion (2010). Annals of DAAAM for 2010 \& Proceedings of the 21st International DAAAM Symposium, ISBN 978-3-901509-73-5, pp 0097, Vienna, Austria 2010

San Andres, L.A. (1993). Dynamic Force and Moments for Short Length Annular Seals, ASME, Journal of Tribology, vol. 115, pp. 61-70

Suciu, C.V. \& Parausanu, I. (1996). A Complet Investigation of unaligned narrow Journal Bearing, Mecanique appliquee, Vol. 41, pp. 99-120 